\title{
Diario di un nefrologo in Africa
}

\author{
Giovanni Battista Fogazzi \\ U.O.C. di Nefrologia, Dialisi e Trapianto di Rene, Fondazione IRCCS Ca' Granda Ospedale Maggiore Policlinico, Milano - Italy
}

\begin{abstract}
This article describes, with the format of a journal, the day by day experience of an Italian nephrologist during one of his humanitarian missions in a hospital in the north of Benin Republic (a subSaharan country of West Africa). Besides the description of his clinical activity and educational programmes (focused on the teaching of the clinical importance of urinary sediment examination), the author supplies a view of the major limitations renal patients face everyday due to the lack of money and adequate diagnostic and therapeutical tools. The author concludes that this unacceptable situation is one aspect of the inequity existing between developed and underdeveloped countries.
\end{abstract}

Keywords: Benin Republic, Nephrological cooperation, Urinary sediment teaching, West Africa

\section{Introduzione}

Ho iniziato a frequentare, come cooperante nefrologo, l'ospedale Saint Jean de Dieu di Tanguiéta, una cittadina nel nord del Bénin (Africa occidentale), nel 1988. Da allora vi torno ogni anno per missioni della durata di due o tre settimane ciascuna. Fin dall'inizio ho affiancato all'attività clinica l'attività di formazione, indispensabile in una nazione in cui il personale e le strutture per la diagnosi e la cura delle malattie renali sono scarsissime. In questo Paese, che conta circa undici milioni di abitanti, i nefrologi attivi sono solo dieci, quasi tutti concentrati nella capitale economica Cotonou; i centri per l'emodialisi sono in tutto quattro; il trapianto di rene è di là da venire, così come la pratica della biopsia renale. In una situazione simile, il coinvolgimento emotivo, oltre che professionale, è inevitabile, e questo mi ha spinto a scrivere il diario di ogni mia missione (1). Qui di seguito è il diario della missione svolta nel 2016.

\section{Prologo}

Torno in Bénin dopo la missione del febbraio 2015 con il progetto di continuare il mio lavoro di formazione in campo

Received: March 6, 2021

Accepted: March 6, 2021

Published online: March 27, 2021

Indirizzo per la corrispondenza:

Giovanni Battista Fogazzi

U.O.C. di Nefrologia, Dialisi, e Trapianto di Rene

Fondazione IRCCS Cà Granda

Ospedale Maggiore Policlinico

Via della Commenda 10

20122 Milano - Italy

giovanni.fogazzi@policlinico.mi.it nefrologico. Un anno fa, il corso sulla sindrome nefrosica a Cotonou e Parakou, organizzato e tenuto con quattro nefrologi del Bénin; quest'anno, un corso di un giorno e mezzo sul sedimento urinario, organizzato con Joseph e Baba - miei exallievi e ora loro stessi docenti - da tenere prima all'ospedale di Tanguiéta e poi a Cotonou. È stato anche in previsione di questo corso che Baba è venuto per un intero mese, lo scorso maggio, nel nostro laboratorio sul sedimento urinario del Policlinico di Milano, uscendo così per la prima volta in vita sua dall'Africa.

Nel corso degli ultimi mesi, però, c'è stata anche la triste vicenda di Romain.

Romain era un ragazzo affetto da insufficienza renale cronica che avevo seguito all'ospedale di Tanguiéta fin da quando era bambino, e che negli ultimi tre anni era in trattamento emodialitico cronico nella città di Parakou, nel Nord del Paese.

La sua salute è andata progressivamente peggiorando, nonostante i due viaggi a Cotonou fatti apposta per curarsi. Il primo per una valutazione cardiologica e il secondo per una riduzione chirurgica della fistola artero-venosa utilizzata per la dialisi. Che la situazione clinica di Romain fosse sempre più delicata lo sapevamo sia io che Martin, che a Parakou aveva accolto Romain a vivere in casa sua, come un figlio. Ma nessuno di noi due poteva immaginare che la fine potesse essere così vicina e che Romain sarebbe morto il 14 gennaio di quest'anno, cioè pochi giorni fa, crollando a terra all'improvviso, pochi minuti dopo il suo rientro da scuola. Una morte sicuramente di origine cardiaca favorita da diverse concause, non ultima la bassa qualità della medicina africana.

Romain, tu e io ci eravamo visti per l'ultima volta nel febbraio dell'anno scorso a Parakou e mi ricordo quell'incontro come se fosse adesso. Tu seduto su un copertone fuori uso e abbandonato in un angolo del cortile (Fig. 1) e io di fronte 


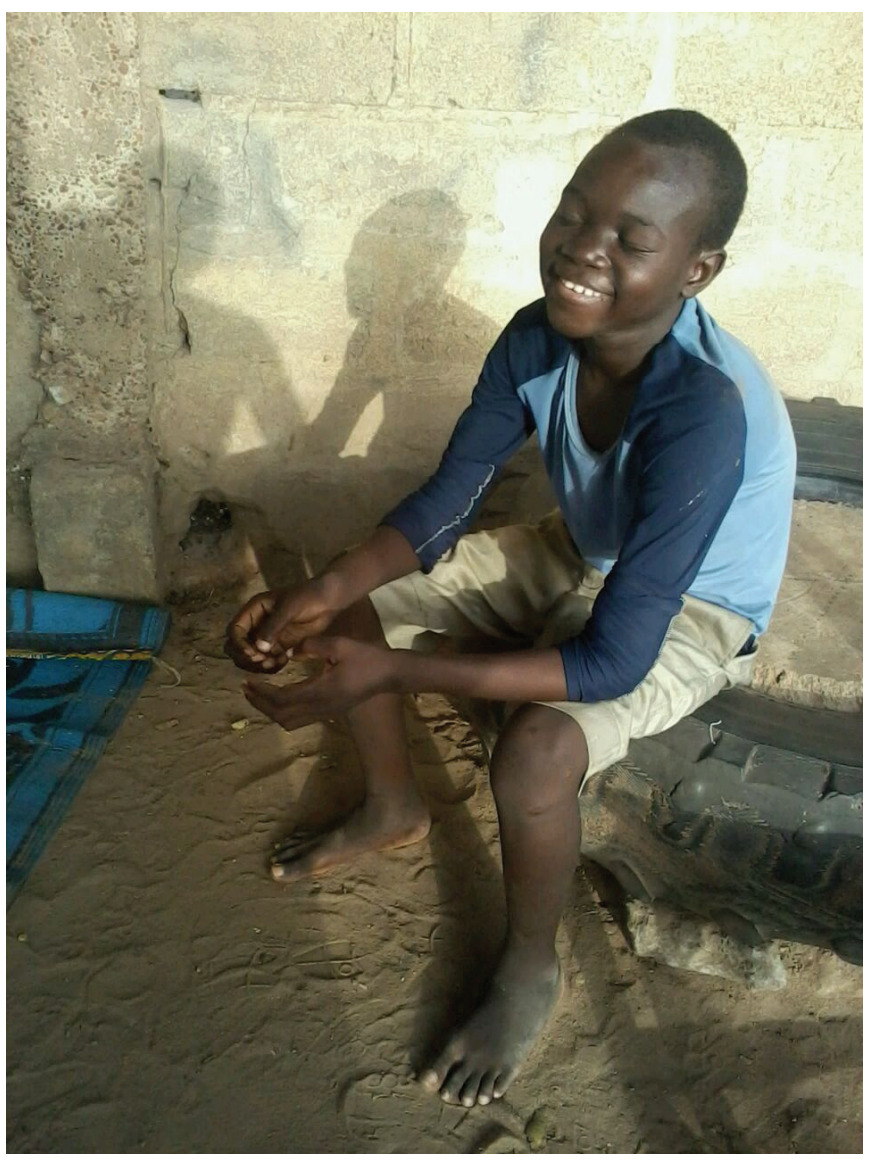

Fig. 1 - Romain nel febbraio del 2015.

a te, ad ascoltare quello che mi raccontavi: a scuola eri tra i più bravi, finito il liceo ti sarebbe piaciuto iscriverti a Ingegneria, ed eri diventato tu il cuoco di casa Martin. Al che io, un po' incredulo, ti avevo chiesto se sapevi cucinare anche il pesce con la salsa di arachidi, e tu mi avevi risposto: "Certo! Guarda però che il pesce e la salsa di arachidi non vanno bene insieme». Mi avevi fatto ridere, ma mi avevi anche fatto capire che oltre ad essere un bravo studente eri anche un bravo cuoco.

Romain, mi mancherai, come a tutti coloro che ti hanno conosciuto e il ricordo di te sarà sempre quello di un ragazzino serio e al tempo stesso dolce, gentile e affettuoso.

\section{In volo tra Parigi e Cotonou Lunedì 25 gennaio}

Mi piace guardare il mondo dall'alto: Parigi, il Mediterraneo e poi l'immenso Sahara. Ma mi piace anche guardare dentro di me e pensare alle cose, anche quelle da fare, e farle: preparare degli esercizi pratici per il corso, sistemare una presentazione non ancora pronta, mettere un po' d'ordine nell'archivio fotografico del computer, raccogliere in un'unica cartella tutte le foto di Romain, ora sparse qua e là.
E adesso che mi sembra di avere fatto tutto e a Cotonou manca solo un'ora e mezza e comincia a scendere il buio e del Sahara qui sotto non si vede più niente, che cosa posso fare?

Riprendo in mano il libro di Paola Mastrocola che mi ha passato Luisa (2) e incomincio a leggere. Dopo poche pagine mi imbatto in un passaggio che mi piace, lo leggo e lo rileggo. È l'asino Raimond, il protagonista del libro, che parla di sé e della sua vita e quel che dice mi piace troppo e lo trascrivo:

"... la mia vita sull'isola ... I pesi che portavo su e giù per le stradine. Come mi piaceva, quanto mi sentivo a posto ... Mi addormentavo stanco morto, e quanto era bello essere stanchi, sentire gli arti che fanno male. Ti sembra di aver fatto il tuo dovere, sai che qualcuno da qualche parte è contento di te, non sai dove ma sai che è così. Ero uno che avevo un posto nella vita".

Bello, vero? Fa venir voglia di darsi da fare e di fare bene, per sentirsi in pace con se stessi e con gli altri ...

Cotonou. Arrivato. All'aeroporto solo un po' di pazienza per il controllo del passaporto, poi il ritiro del bagaglio è veloce. Fuori ci sono Bienvenu, Olivier, Martin e Marcellin.

Bienvenu, come Romain, è un ragazzo che ho conosciuto come paziente a Tanguiéta quando era ancora bambino. Nel corso degli anni la sua malattia renale si è aggravata fino alla necessità di sottoporsi all'emodialisi cronica. Per tale motivo è stato trasferito a Cotonou, dove vive e studia, con il sostegno economico del nostro gruppo "Noi di Tanguiéta".

Sorrisi, abbracci, saluti. Poi in un quarto d'ora, con il taxi di Henry arriviamo all'AMCES (Association des Oeuvres Médicales Privées Confessionnelles et Sociales au Bénin), una associazione multiconfessionale che organizza e ospita convegni e corsi di argomento sanitario e mette a disposizione camere per docenti e allievi, e che considero la mia casa, qui a Cotonou.

Dopo pochi minuti rimaniamo soli, Bienvenu, Olivier e io. Andiamo a cena. Questa volta a pochi metri da qui, dall'altro lato della strada, in un ristorante "africano", diverso da quello vicino allo stadio dove siamo stati in precedenza, un po' troppo europeo e anche un po' caro. Ordino riso e tacchino: non sono tanto buoni, ma forse anche perché è un po' tardi. Del resto, non è nemmeno così importante: conta di più sentire cosa mi raccontano Bienvenu e Olivier. In realtà nessuno dei due parla moltissimo, anzi bisogna un po' tirargli fuori le parole con le pinze. Cerco di sapere da entrambi come va l'università, ma ci capisco poco. "Sarà il sistema, che è diverso dal nostro" mi dico e cambiamo argomento. Bienvenu sta abbastanza bene, la dialisi non sembra dargli grossi problemi, gioca ancora a calcio e di tanto in tanto fa dei piccoli lavori per racimolare qualche soldo. Mi sembra abbastanza contento, e anche Olivier. Ma per lui è più semplice perché è sano e ha una famiglia che si occupa di lui, anche se lontana, a Tanguiéta. 
E adesso che ci siamo rivisti, abbiamo chiacchierato un po' e cenato insieme, è ora che andiamo a dormire visto che questa mattina mi sono alzato molto presto.

Bienvenu e Olivier, buonanotte e a presto.

\section{Tanguiéta Martedi 26 gennaio}

Sera. Arrivato a destinazione, qui, nell'altro ospedale della mia vita. Avrei dovuto atterrare a Ouagadougou in Burkina Faso, avevo già il biglietto, e arrivare a Tanguiéta da una strada più breve e confortevole. Tuttavia, ho dovuto cambiare programma a causa di un attentato di Al Quaeda, che il 16 gennaio a Ouagadougou ha provocato ventisette morti.

Sono partito da Cotonou questa mattina, però non alle 7 e non con il bus dell'ATT come le ultime volte ma su un'auto dell'ospedale, che si trovava già a Cotonou per portare a Tanguiéta fra Nicolas e fra Marcel di ritorno da Roma, più una quantità incredibile di merci di tutti i tipi, a occupare ogni centimetro cubo di spazio.

Durante tutto il viaggio, io privilegiato perché sono seduto davanti, in business class, mentre i due frati, dietro in economy, stretti tra scatole, scatoloni, borse. lo di fianco all'autista Cyril, con il quale non avevo mai viaggiato prima.

Cyril, che sulla strada poco fuori Cotonou andava a tutta birra in mezzo a un traffico allucinante - tanto che a un certo punto ho pensato: "Altro che attentato jihadista, io qui ci lascio le penne per molto meno" e me la sono fatta sotto per un bel po' ... - fino a quando, a mano a mano che salivamo verso Nord, il traffico ha incominciato a diradarsi e Cyril, forse anche per la stanchezza, a calmarsi. «Cyril, scusa se te lo dico, guidi sicuro di te e si vede che sei abile, ma onestamente spero sia la prima e ultima volta che vengo con te. Guarda, quasi quasi era meglio l'autobus.»

Nonostante la partenza sprint, il viaggio è stato lungo anche questa volta: siamo partiti che era quasi mezzogiorno e siamo arrivati poco dopo le 21. Pranzo veloce a Dassa, come già altre volte nel ristorante di madame Kpanou, e poi via di nuovo su su verso Nord, sempre più dentro al "paese profondo".

Lungo la strada ho avuto molto tempo per pensare e notare che:

- in lontananza, c'è foschia, quasi nebbia. È l'harmattan, mi dicono. D'accordo, è il periodo e ci sta. Però cosa c'entra il vento che soffia dal deserto del Sahel se qui è tutto fermo e non si muove foglia?

- A mano a mano che si sale verso Nord, molte le moschee che si intravedono, anche tre o quattro in villaggi piccoli piccoli. Per lo più moschee minuscole e dimesse ma segno inequivocabile della diffusione capillare dell'Islam.

E alla fine eccoci all'ospedale. Parcheggiamo giusto dietro la casa dei frati. Non facciamo in tempo a scendere che già vengono ad accoglierci e a prendere i bagagli. C'è anche suor
Carmen, con il suo bel sorriso: "Ciao Carmen, che piacere vederti!» e il suo sorriso diventa ancora più bello.

Cena veloce nel refettorio, in compagnia di fra Fiorenzo, amichevole e pieno di energia come sempre. Saluti e scambio di informazioni e poi via verso la mia camera, la n. 9, con le mie due valigie gentilmente portate per me da due novizi.

\section{Tanguiéta Mercoledi 27 gennaio}

La prima cosa fatta questa mattina è stata portare a suor Carmen le foto dei suoi affreschi del monastero di Materi, dove lei è stata per qualche anno e del Saint Jean de Dieu, con l'album (fatto a mano dalla legatoria Conti Borbone di Milano) dove mettercele e lo stick di colla per attaccarcele. II tutto in un bel sacchettino elegante.

Grande sorpresa da parte di suor Carmen e grande successo: «Tita, è il più bel regalo che abbia mai ricevuto in tutta la mia vita!». "Carmen, se sei contenta tu, puoi facilmente immaginare quanto lo possa essere anch'io, di sicuro non meno di te!» E poi il programma di vederci alla sera e incollare le foto insieme ...

Insomma, proprio un bel modo di incominciare la giornata. E adesso, al lavoro:

Medicina. Dieci pazienti con problemi renali, già inquadrati però da Séraphin, che è arrivato qui lunedì, per la sua periodica missione. Séraphin è un nefrologo che lavora presso l'ospedale di Parakou, la principale città del Nord del Bénin. Ogni due mesi effettua una missione nefrologica della durata di tre giorni presso l'ospedale di Tanguiéta, dove il nefrologo manca. Anche queste missioni sono sostenute con fondi raccolti dal nostro gruppo "Noi di Tanguiéta".

Pediatria. Due soli pazienti, di cui una è Penagui, di 14 anni circa, che conosco bene perché avevo incominciato a curarla, con Guy, nell'ottobre 2014 e poi l'avevo rivista, sempre qui, nel febbraio scorso. Dunque, adesso la sua situazione è la seguente: insufficienza renale cronica ormai da dialisi (creatininemia 10.8 e urea $180 \mathrm{mg} / \mathrm{dL}$ ) + malattia cardiaca. Casa in un villaggio vicino a Materi, il padre deceduto, e la madre che vive in pianta stabile qui, sotto i manghi del grande cortile dell'ospedale (Fig. 2), con altri due figli, bambini di 3-4 anni. Da quando Penagui è qui, la mamma non è più tornata al villaggio, per poter stare vicino alla figlia malata. Vivendo come? Facendo dei lavori di tanto in tanto in paese e dormendo dove ho già detto, sotto i manghi.

Quindi, primo giorno di lavoro e a fine mattina già il primo caso su cui riflettere: che fare di Penagui, ragazzina di 14 anni, ricoverata in ospedale da almeno 16 mesi, senza mezzi e senza casa, affetta da insufficienza renale grave e che da noi, in Italia, sarebbe già curata con la dialisi? Purtroppo non sono nuovo a situazioni del genere e adesso so anche cosa fare: lasciare che i pensieri trovino la loro strada dentro di me, poi parlarne con i medici che seguono Penagui e anche con suor Carmen che la conosce bene, e insieme prendere una decisione. 


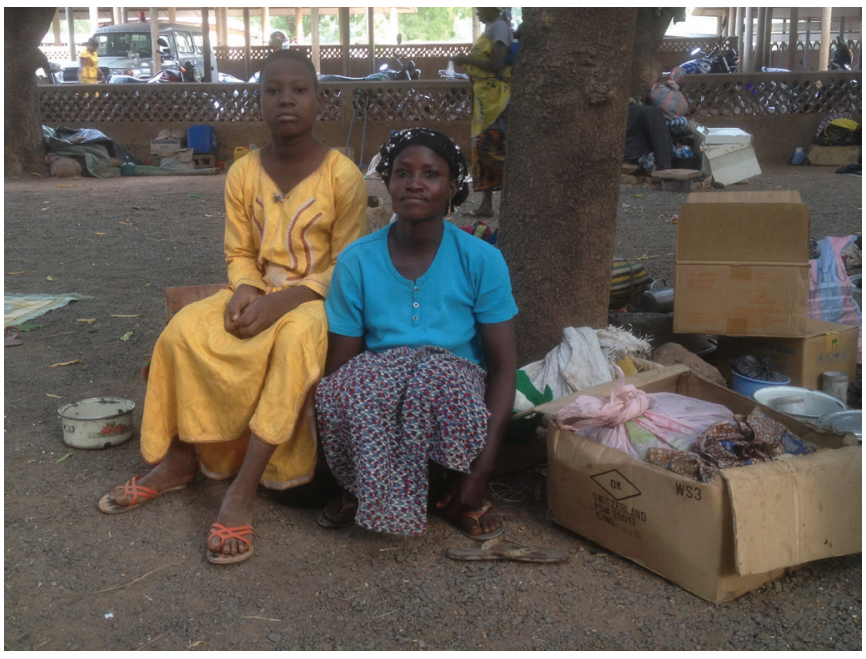

Fig. 2 - Penagui (a sinistra) con sua mamma nel grande cortile dell'ospedale, tra i manghi. Scatoloni, sacchetti e pentole sono tutto ciò di cui dispongono.

Ambulatorio. Sei pazienti, tra cui Barri Fati, che conosco da alcuni anni. Per lei la necessità della dialisi si sta a poco a poco avvicinando e per questo Fati è giù di morale e mi guarda con aria sconsolata. Ma per lei la situazione è molto meno complicata che per Penagui. Perché? Perché Fati ha 44 anni, è attiva, e ha un lavoro, commerciante, che le permette di essere autonoma. Inoltre Séraphin è già riuscito a ottenere per lei la presa in carico da parte dello Stato, per cui quando dovesse cominciare la dialisi non dovrebbe pagare niente. Unici due ostacoli: 1) Fati dovrebbe andare a vivere a Parakou, perché la dialisi per ora c'è solo lì. 2) L'allestimento della fistola-arterovenosa, che serve per poter fare la dialisi e che può essere fatto solo privatamente a Lomé, costa parecchio. Parliamo di tutto questo con Fati, che ascolta attentamente ma sempre con aria sconsolata. A un certo punto, però, le dico: "Senti, Fati, tu sei una persona in gamba e sono sicuro che potresti avere una vita di buona qualità nonostante la dialisi due volte alla settimana. Pensaci ... se accetti di trasferirti a Parakou, noi copriremo le spese per la fistola, viaggio e soggiorno a Lomé. Pensaci e facci sapere, io sono qui fino a mercoledì prossimo». Quando esce dall'ambulatorio Fati è ancora perplessa, però forse un po' meno di quando è entrata.

E infine, piacevole sorpresa di fine giornata. Quando dopo cena ho riprovato la pressione arteriosa a Béatrice, una paziente che era venuta in ambulatorio nel pomeriggio, e le ho chiesto che età avesse, mi ha detto: "Dottore, sono nata nel 1953 e quindi quest'anno compio 63 anni».

«Come me!» ho risposto. "E in che mese e giorno sei nata esattamente?» «Il 18 giugno.»

Incredibile, stesso anno, mese e giorno in cui sono nato io! Con una differenza non da poco, però: Béatrice è nata ad Abomey e io nel lato fortunato del pianeta Terra ...

\section{Tanguiéta Giovedi 28 gennaio}

Tutto il giorno a lavorare per il corso. Dapprima in laboratorio con Joseph e Baba, poi da solo, e poi di nuovo dopo cena, qui in camera. Le cose di cui occuparsi sono tante. In primis gli interventi, miei e di Joseph e Baba - controlla, correggi, taglia, lima, suggerisci, ecc. ecc. - e poi gli aspetti organizzativi, di cui in realtà si è occupato molto efficacemente Joseph. Adesso, finalmente, sembra tutto ok, e quattro dei dodici iscritti sono già arrivati e hanno cenato nella sala che sta di fianco alla mensa di noi cooperanti, preparata a puntino da suor Felicité. Li ho visti e sono entrato a salutarli e a presentarmi. Mi sembrano simpatici.

In Medicina è ricoverato un ragazzo di 20 anni, Daniel, per anuria quasi completa, cioè niente pipì. Fatta l'ecografia al volo: dilatazione molto severa dei reni e degli ureteri e vescica quasi vuota e con pareti molto ispessite. Un caso per i chirurghi. Intanto però ho cercato di raccogliere qualche informazione: si chiama Daniel (Fig. 3), viene da un villaggio della savana, parla solo la lingua berba, e ha dolore al basso ventre già da un po' di tempo. A gesti, ho cercato di fargli capire che domani torniamo a vederlo. Così magari questa sera si sente meno solo.

Oggi pomeriggio, una suora del Burkina Faso che avevo curato qualche anno fa è venuta in laboratorio, del tutto inaspettatamente, per portarmi un regalo, anzi due: un sacchetto di arachidi zuccherate (come avrà fatto a sapere che mi piacciono tanto?) e un barattolo di marmellata di arance.

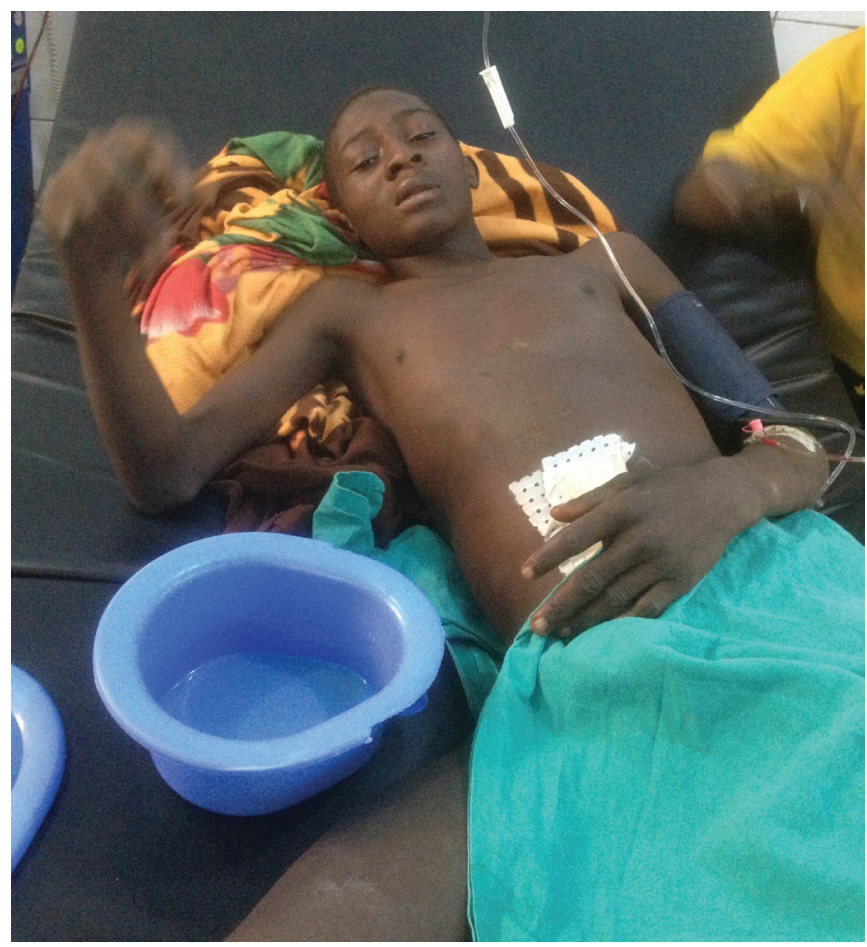

Fig. 3 - Daniel. 
Ancora una volta la conferma che anche gli africani sanno donare.

\section{Tanguiéta Venerdì 29 gennaio}

Corso. noi tre, i docenti, più dodici iscritti, provenienti da Zinvié (poco sopra Cotonou, molto lontano da qui), Djougou, Parakou, Nikki (che mi intriga perché è vicina al confine con la Nigeria), Bembèrèkè (intrigante pure questo villaggio perché è sulla strada per Malanville dove passa il Niger, e io una volta o l'altra ci voglio andare a vederlo questo fiume che attraversa non so quanti Paesi dell'Africa occidentale e si porta dietro chissà quante e quali storie) e da Tanguiéta. Tecnici, biologi, studenti di medicina e medici.

Lavorato dalle 9.30 alle 17.30 , con una pausa pranzo (menu ottimo) e una pausa-caffè a metà pomeriggio.

Livello dei partecipanti (conoscenza + esperienza) piuttosto basso, però pongono molte domande appropriate e hanno molto interesse, senza il quale non si va da nessuna parte.

Per quanto riguarda noi tre, i docenti: Joseph: perfetta la sua presentazione. Baba: si è perso un po' nella parte pratica sul microscopio in contrasto di fase, però per entusiasmo, voglia di fare, e fare bene, ci siamo. lo: fatte quattro presentazioni e parlato, in tutto, per circa cinque ore, senza contare le sessioni di discussione.

Quindi per il corso questa sera sono molto soddisfatto.

Daniel: oggi è stato trasferito in Chirurgia. Sono passato a salutarlo con Séraphin. Mi fa molta tenerezza.

\section{Tanguiéta Sabato 30 gennaio}

Corso n. 1 finito. Con una sezione extra di due ore dopo pranzo per poter finire i tredici esercizi pratici di refertazione: noi docenti presentiamo dei referti contenenti i dati dell'esame delle urine e gli allievi devono interpretarli cercando anche di capire quale tipo di malattia dell'apparato urinario ci può essere dietro.

Per quest'ultima parte ci siamo trasferiti in laboratorio, seduti attorno al tavolo (Fig. 4), perché siamo rimasti in sette. Cinque dei partecipanti, infatti, hanno dovuto prendere l'autobus subito dopo il pranzo per tornare a casa.

Impegno e interesse notevoli, con molta interattività.

Quindi, conclusione dei lavori alle 15.30. Ancora una volta, pur sapendo che siamo solo all'inizio dell'inizio, sono fiducioso, perché le persone che ho incontrato mi sono sembrate motivate.

Dopo il lavoro, le foto e i saluti, ho voglia di rilassarmi e di uscire un po' dall'ospedale.

Vado a piedi fino alla piazza del mercato che è sempre un po' affollata, anche se non come al lunedì, che è il giorno ufficiale del mercato di Tanguiéta. Appena superato l'ingresso principale, ho incontrato Aliatou, la moglie di Baba, seduta al suo banchetto dove vende sacchetti e scatolette

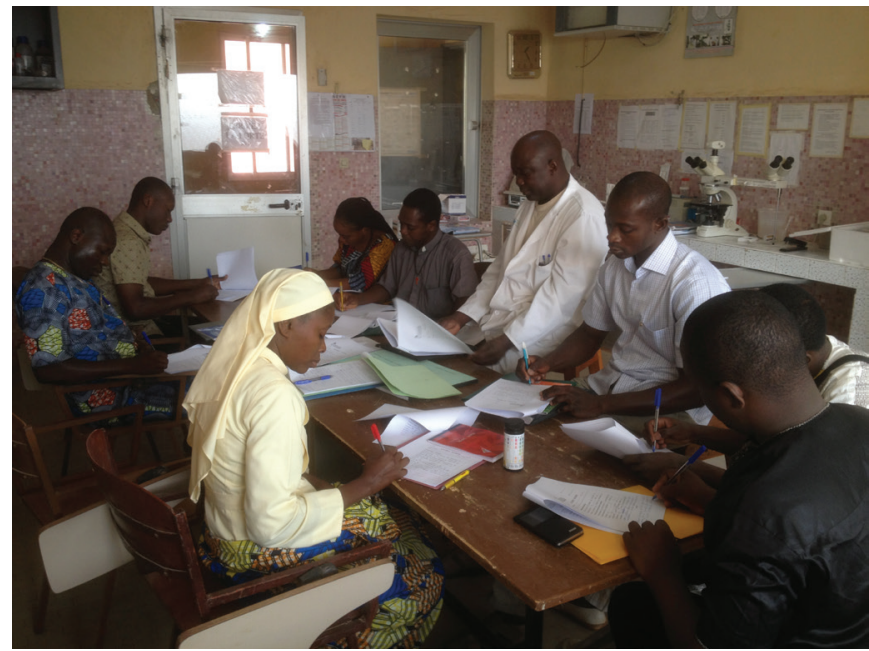

Fig. 4 - Esercizi di refertazione a fine corso sul sedimento urinario.

di tutti i generi, in compagnia dei suoi quattro figli: Saef e Alim (maschi), Fatima, già velata da brava musulmana, e Chakira (bambine). Aliatou saluta sorridente; salutano anche i bambini, però senza sorriso, un po' intimiditi. Ricambio il saluto, quattro chiacchiere e poi incomincio a girare per le bancarelle.

C'è di tutto: stoffe africane e indumenti, per lo più usati, scarti - mi vien da pensare - di noi europei, che arrivano fin qui attraverso chissà quali canali; verdure e cereali; pesce, che non ho mai capito come venga conservato, sembra fritto $\mathrm{ma}$ in realtà è scuro, quasi nero, e ha un odore forte e acre che ti fa passar la voglia; pentole e pentolini smaltati dai disegni colorati bellissimi, che arrivano dalla Nigeria, che a me piacciono molto, ma ancora di più mi piacciono quando sono vecchi, usati e ammaccati. $E$ in mezzo a tutta questa merce, la gente. Mi sorprende vedere quante bambine e bambini ci sono, probabilmente figli delle venditrici. Mentre passo li guardo, sorrido e saluto con la mano, mi viene voglia di fotografarli. Mi guardano a loro volta, ma si intimidiscono: molti, soprattutto le bambine, si coprono il viso (Fig. 5), si nascondono le une dietro le altre, alcune addirittura scappano.

Dopo cena incontro con fra Fiorenzo nella mensa dei cooperanti: bilancio del corso e nuovi progetti. Parliamo anche di Daniel, che lui ha operato questa mattina per cercare di rimuovere l'ostacolo che blocca il flusso dell'urina. Purtroppo, non ha potuto fare niente perché gli ureteri e la vescica sono inglobati in un piastrone granulomatoso, da probabile schistosomiasi. Qui, come in altri Paesi dell'Africa, lo schistosoma haematobium, un parassita che si insedia nella vescica, è endemico e, se trascurato, può causare danni gravissimi irreversibili, come nel povero Daniel. La sua situazione sarebbe difficile anche in Italia, tuttavia curabile, anche se attraverso un percorso lungo e complesso. Qui invece non si può fare niente e anche il destino di Daniel, che poco prima di cena sono passato a salutare, è segnato. 


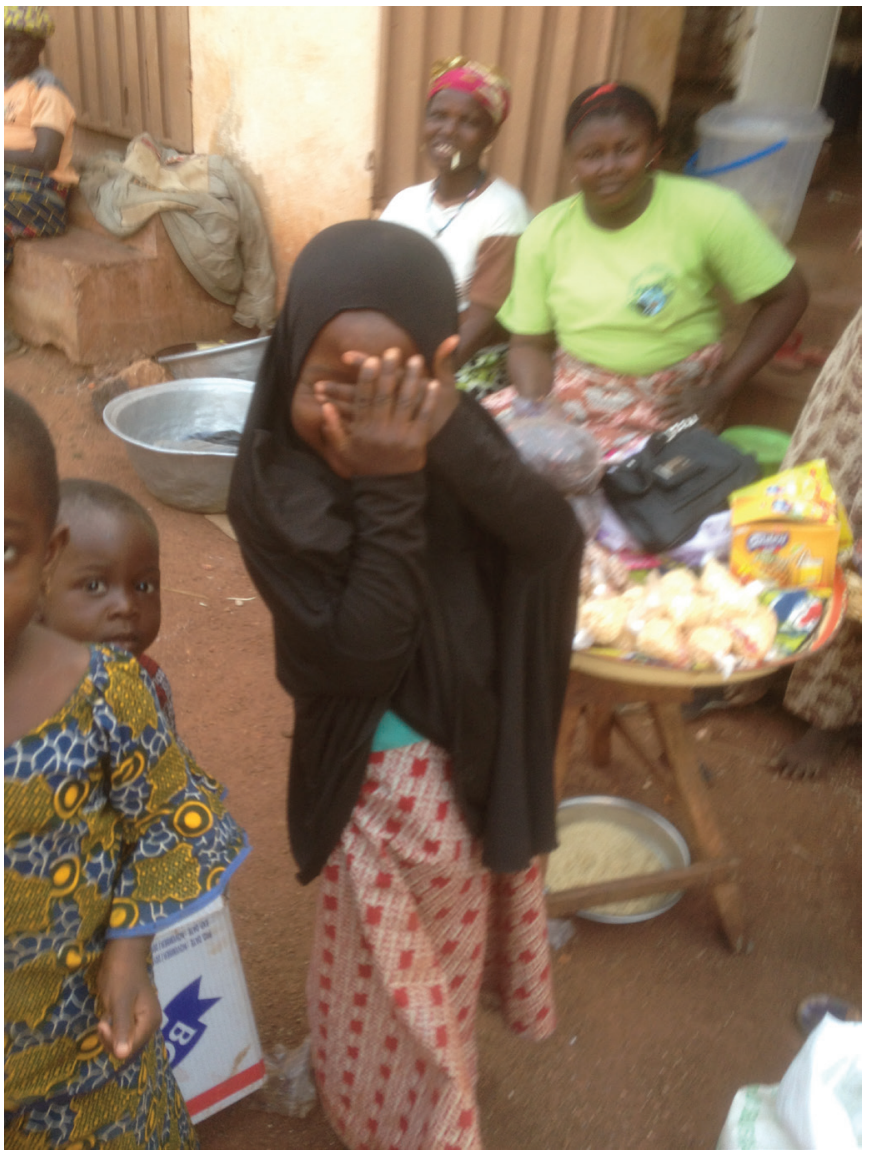

Fig. 5 - Al mercato di Tanguiéta.

\section{Tanguiéta Domenica 31 gennaio}

Questa mattina giro di visite in Medicina con William, medico giovane e gentile, ai malati con problemi renali, che in questi due giorni abbiamo trascurato a causa del corso. Nella notte è deceduta la paziente con insufficienza renale molto grave, al di là di ogni speranza. Si chiamava Natchabo Salamatou e aveva 35 anni.

Per il resto della mattina e fino alle quattro del pomeriggio, sono da solo, nella mensa dei cooperanti a risistemare i file per il corso di Cotonou. Solo dettagli, ma utili per migliorare.

E poi di nuovo in giro per Tanguiéta come ieri. Arrivato all'incrocio, giro a sinistra e mi incammino per la strada che porta alla "Galleria d'Arte" di Assan Gueye, un musulmano amico di Baba, dove vado tutti gli anni ma dove non ho mai comprato niente. Me lo fa notare ogni volta anche lui. Ma Assan, è colpa mia se io cerco cose vecchie e tu di vecchio non hai niente o quasi? Tu trovami la cosa giusta e vedrai che, nonostante tutte le raccomandazioni di Luisa e Nick di piantarla di comprare cose inutili, io quella cosa te la compro. Quindi, Assan, è vero che non compro mai niente da te, ma la cosa dipende più da te che da me.

E mentre torno verso l'ospedale incontro Samson, il fratello di Bienvenu, che vive qui a Tanguiéta facendo il falegname. Insiste perché vada con lui a casa sua, a vedere il suo laboratorio. Ok, andiamo: camminiamo per una ventina di minuti, fino ad arrivare dall'altra parte del paese, non lontano da dove abitavano un tempo Robert, bravissimo e motivato tecnico di laboratorio, e Outa $\mathrm{Nda}$, il guaritore tradizionale.

Entriamo nel cortile, dove c'è un mobile non ancora finito. Entriamo in casa, una sola stanza dove Samson fa tutto. Ha anche una piccola collezione di oggetti inutili, come il carapace di una tartaruga e dei sassi dalla forma strana, più alcuni animali di legno fatti da lui, abbastanza belli. Al muro è appesa una grande fotografia di suo papà, che assomigliava molto a Bienvenu.

Chiedo a Samson dov'è il suo laboratorio:

"Qui» risponde, «in cortile.»

«E gli attrezzi?»

"Qui anche loro" risponde, indicandomi una cassetta di legno. Guardo dentro: una sega, un paio di martelli, due lime, una pialla, un trapano a mano, dei cacciaviti e, mi sembra, niente altro.

"Samson, tutto qui?!»

«Si, Dottor Tita.»

Al che penso due cose:

- Samson, come cavolo speri di finire quel mobile?

- Adesso ho capito perché hai insistito tanto per farmi venire fin qui: speri che il "bianco" ti procuri qualche arnese, possibilmente elettrico.

Dopo cena: incontro con suor Martine e suor Carmen per fare il punto sui progetti in corso e consegnare i soldi che abbiamo raccolto per il loro finanziamento.

\section{Tanguiéta Lunedì $1^{\circ}$ febbraio}

Mattina. Ambulatorio: cinque pazienti già conosciuti, tra i quali di nuovo Barri Fati, a cui ho rispiegato, cercando di incoraggiarla, perché abbiamo pensato di sostenerla per la fistola artero-venosa per la dialisi. Poi, nel pomeriggio in laboratorio a preparare con Joseph e Baba il corso di Cotonou. $\mathrm{Ci}$ abbiamo messo un po' ed è stato piuttosto faticoso, ma adesso dovrebbe essere tutto a posto.

Prima di cena appuntamento nella scuola annessa alla Pediatria con suor Carmen, Penagui e sua mamma, per spiegare loro che ci farebbe piacere aiutarle -30 euro al mese affinché possano affittare una stanza in paese e non debbano più vivere sotto i manghi nel grande cortile dell'ospedale. Sappiamo che Penagui non vivrà a lungo - organizzare il trasferimento suo e di tutta la famiglia a Parakou per la dialisi cronica sarebbe impossibile e le esperienze avute finora con altri giovani pazienti sono state tutte molto scoraggianti - ma che almeno gli ultimi mesi della sua breve vita possano essere vagamente decenti. Dato che la mamma di Penagui non sa il 


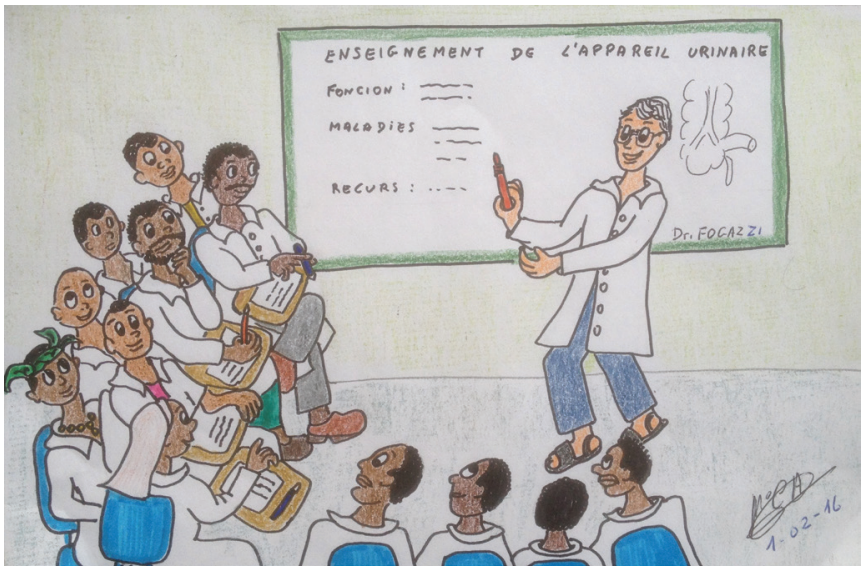

Fig. 6 - Il corso sul sedimento urinario visto da suor Carmen.

francese, il colloquio si è svolto con un giro a tre: suor Carmen e io ci rivolgiamo a Penagui in francese e lei traduce a sua mamma in berba e viceversa. Un fatto comune da queste parti, ma che ogni volta mi colpisce un po'.

Finito l'incontro, suor Carmen mi regala un suo disegno, che ritrae me mentre insegno al corso (Fig. 6). Carmen, inutile dirti che questo tuo disegno, come tutti gli altri del resto, $\mathrm{mi}$ piace molto e mi fa un enorme piacere. Tu artista e, in più, mia amica.

\section{Tanguiéta Martedì 2 febbraio}

Durante il giro in Medicina due scoperte:

- William, che in questi giorni aveva gestito in prima persona i pazienti nefrologici ricoverati e al quale mi ero già un po' affezionato, da questa mattina è trasferito all'ambulatorio di Porga, a 40 chilometri da qui sul confine con il Burkina Faso, dove finora c'erano solo infermieri. Anche se capisco le ragioni del trasferimento, mi dispiace: William è una brava persona e un medico affidabile. Ma forse è proprio per questo che l'hanno voluto lassù, in piena savana e in prima linea.

- Da alcuni mesi i pazienti ricoverati con febbre devono misurare la temperatura corporea con un termometro personale, che viene loro venduto dall'ospedale a 700 franchi CFA (poco più di $1 €$ ). La ragione: prevenire la trasmissione di malattie contagiose. Visto quello che è successo con il virus di Lassa a fine 2014 mi sembra una decisione molto saggia.

Abbiamo recuperato finalmente l'ecografo portatile che nei giorni scorsi sembrava sparito nel nulla e ricominciato quindi a fare ecografie renali al letto del malato, insieme a Séraphin e Pauline, studentessa di Medicina di Bruxelles, che è qui per sei mesi.

E finalmente consegnati al Deposito Farmaceutico dell'ospedale i farmaci per i pazienti nefrologici che avevo comprato prima di partire. A parte l'Enalapril da $5 \mathrm{mg}$, degli altri non era rimasta nemmeno una compressa, quindi: ben fatto.

Daniel. Le medicine che gli abbiamo dato in questi giorni come ultima speranza non hanno per ora migliorato la situazione. Persiste l'assenza quasi completa di diuresi e gli esami della funzione renale sono di conseguenza peggiorati. Vado a trovarlo due volte al giorno e ogni volta, a gesti, cerco di fargli coraggio.

\section{Tanguiéta Mercoledì 3 febbraio}

Mattina. Dopo la riunione con i medici dell'ospedale e il giro in Medicina, visita a Daniel con l'amico e collega Diombo. Ragionato di nuovo su cosa possiamo fare per lui.

Poi in moto, presa in prestito ancora una volta da Lucien, a Materi, con Pauline sul sedile posteriore. Gli ultimi 15 chilometri di pista sempre affascinanti, anche se il paesaggio in questo periodo dell'anno non è certo quello, tutto verde e rigoglioso, di ottobre.

Monastero di Materi. Turnover a 180 gradi delle suore: Antonia è stata trasferita a dirigere la Casa di Ouagadougou in Burkina, Marta è stata trasferita in Spagna; al loro posto sono arrivate, dalla Spagna, Carmen ("Carmen 2") e Anna. Invariate invece le due suore africane, Adrienne (del Bénin) e Florence (del Burkina).

Suor Anna. Lì per lì mi era sembrata una faccia nuova, ma poi chiacchierando mi sono ricordato un po' alla volta, e lei con me, che ci eravamo conosciuti già 15 anni fa, quando lei viveva con le altre suore in ospedale a Tanguiéta e insieme con suor Antonia - avevamo lavorato al progetto per costruire un nuovo edificio per la scuola di Yehoungou. Sorpresa reciproca e stupore: vedi un po' che giri fa la vita, e quanti fatti e persone si rischia di dimenticare ...

Poi, visita guidata al monastero per Pauline che non è mai stata qui, e durante la quale ne ho approfittato per fotografare tutti gli affreschi fatti qui a suo tempo da suor Carmen, e poter aggiungere poi le foto nel mitico album che le ho portato. E infine, pranzo, come sempre ottimo e in un'atmosfera ospitale e serena, anche se le suore sono in parte "nuove".

Sulla via del ritorno, lungo la pista, stop a un minuscolo villaggio. C'è un pozzo e tutto attorno tante ragazze con le loro taniche da riempire. A un certo punto, una di loro esce dal gruppo si mette sulla testa la sua tanica da 25 litri piena d'acqua e imbocca un sentiero che va verso la collina. La seguo e dopo pochi metri le chiedo dove va. "Al mio villaggio, che è là in fondo", mi risponde, a circa un chilometro da dove siamo. Le chiedo di fermarsi e di passarmi la tanica. A fatica me la metto sulla testa e incomincio a camminare, ma dopo pochi metri non ce la faccio già più e sono costretto a restituire la tanica alla ragazza, che se la rimette in testa ridendo. 
Rido anch'io, ma meno di lei, vuoi per la magra figura, vuoi per il pensiero che non deve essere uno scherzo, nemmeno per una giovane e forte ragazza africana - che avrà sì e no 18 anni - farsi un chilometro a piedi con 25 chili sulla testa. E magari non una ma più volte al giorno, per portare a casa una cosa indispensabile come l'acqua.

Rido un po', sì, ma dentro di me provo anche vergogna perché penso a tutte le "balle" che noi del lato fortunato del pianeta Terra abbiamo, e non siamo mai contenti di niente, e l'acqua ci arriva in casa, senza la minima fatica, e molto spesso la sprechiamo.

Tornato in ospedale, vado da suor Carmen a dirle che ho completato le foto dei suoi affreschi e intanto ne approfitto per farci fare qualche foto insieme, noi due, da amiconi e sorridenti.

Oggi è il mio ultimo giorno qui, lo so io e lo sanno anche gli altri. E così poco prima di cena fa la sua comparsa Fati, che mi viene a salutare e mi porta, in regalo, una stoffa africana, gesto che mi commuove. Idem per la telefonata di Béatrice, la mia "coscritta", che mi saluta e mi augura buon viaggio per domani.

Anch'io passo a salutare, in primis Daniel e poi gli altri: Marina, Tiziana e Mario, anche loro cooperanti italiani affezionati da anni a Tanguiéta e ovviamente le suore. Che mi fanno una sorpresa chiamando per me al telefono suor Cristina, con la quale ho gestito i nostri progetti per anni e che adesso è di stanza in Spagna, a Burgos, da dove, quasi piangendo, mi dice che ha molta nostalgia dell'Africa e che vorrebbe tornare qui.

\section{Cotonou Giovedì 4 febbraio}

Partiti questa mattina alle 6.50, anche questa volta con una vettura dell'ospedale. Sì, perché Joseph, Baba e io siamo in missione ufficiale, in quanto docenti al corso che si replicherà domani e sabato a Cotonou, e quindi abbiamo diritto a un'auto tutta per noi. Un autentico lusso.

All'ultimo momento si è aggiunto al gruppo Séraphin, che però è sceso prima, a Bohicon, per andare non ho capito dove. Séraphin ha imbarcato nell'auto oltre alla valigia anche dei sacchi di mais, miglio e riso - comprati dalla sua fornitrice di fiducia, nonché sua e mia paziente Barri Fati - e due scatole di cartone tutte bucate, con dentro dei piccioni vivi: un altro esempio dell'Africa rurale, che fa capolino anche quando meno te l'aspetti.

Al volante Roger, socievole e soprattutto prudente, che conosco da tanti anni e con il quale ho già fatto non so quanti viaggi, compreso quello con il guaritore Outa $\mathrm{Nda}$, quando nel 2002 siamo andati nella savana a cercare la famosa radice che cura i reni.

Come già all'andata, io "il bianco" davanti, e gli altri tre dietro. Durante il viaggio abbiamo chiacchierato molto di più che all'andata. Argomenti: un po' di tutto, compresa la magia africana, che era già un po' che non saltava fuori. A ogni episodio che mi raccontano dico che non ci credo, che sono tutte storie inventate, e rido. lo rido ma loro no, sono serissimi: qui alla magia ci credono tutti, compreso il razionale ed emancipato Joseph, che due giorni fa mi ha persino mandato su WhatsApp un video che mostra che a Natitingou ci sarebbe un albero che si rimette in piedi da solo e nel giro di pochi secondi ogni qualvolta una ruspa cerca di buttarlo giù perché è cresciuto troppo e per giunta in mezzo a una strada. lo non so, continuo a non capire perché gli africani persistano nel loro attaccamento a queste credenze senza, apparentemente, nessun cambiamento nonostante il passare del tempo e l'arrivo della tecnologia anche qui.

Albert Schweitzer (1875-1965), che nel 1913 ha fondato l'ospedale di Lambaréné (Gabon) dove ha speso il resto della vita, ha descritto gli stessi identici comportamenti quasi cento anni fa (3).

Tra Savalou e Dassa, breve sosta al cimitero di Logozohé per un saluto alla tomba di Guy, che in questo villaggio era nato (Fig. 7).

Guy è stato per anni il primario del reparto di Pediatria dell'ospedale di Tanguiéta e per me, oltre che un bravissimo e motivato collega, anche un caro amico. Nel novembre del 2014 è deceduto in seguito a una infezione dovuta al virus di Lassa, che è endemico in diverse aree dell'Africa occidentale.

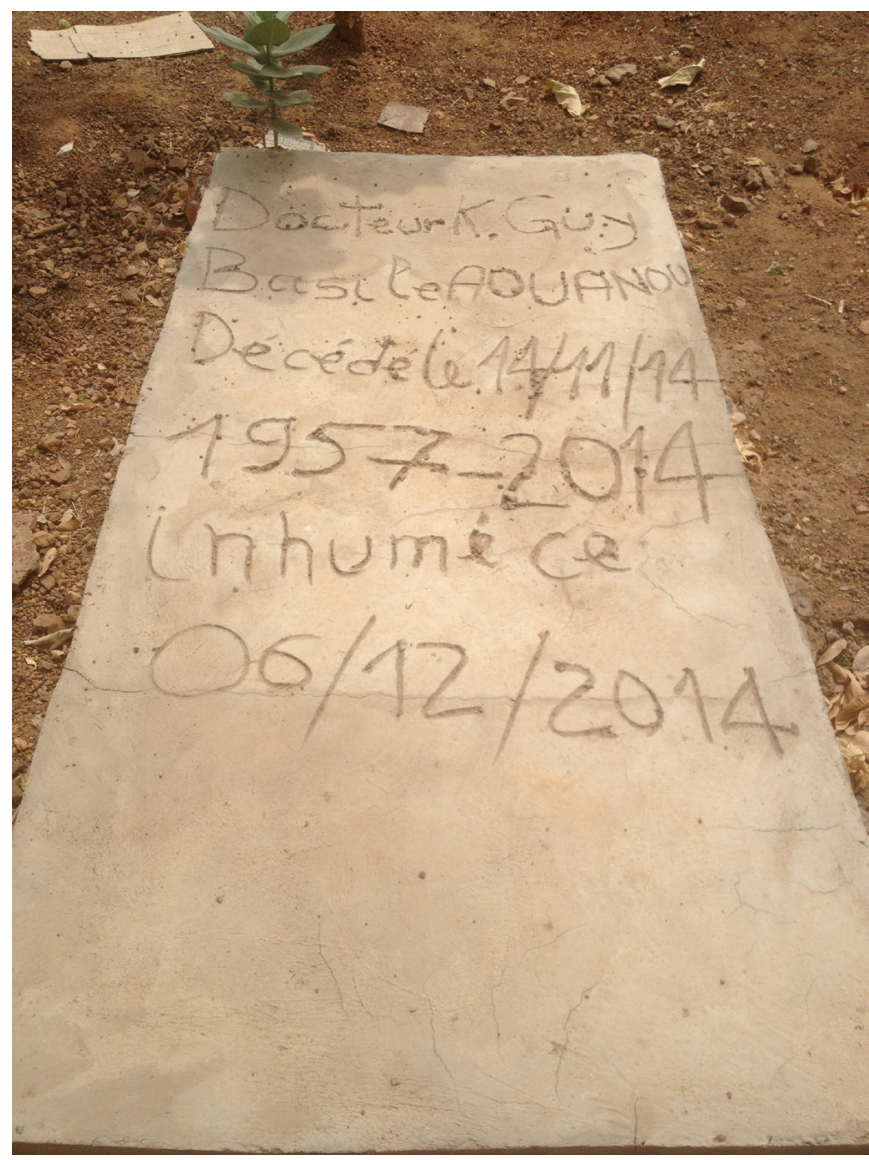

Fig. 7 - La tomba del Dr. Guy al cimitero di Logozohé. 
Quando risaliamo in auto Joseph mi dice che anche fra Fiorenzo si ferma a salutare Guy ogni volta che si trova a passare di qui. Guy, hai davvero seminato bene durante la tua vita, e le persone che ti hanno conosciuto si ricordano di te e lo dimostrano.

Arrivati a Dassa, ci fermiamo al solito ristorante per il pranzo. Oggi l'ambiente è ravvivato da un venditore ambulante che ad alta voce reclamizza un prodotto che a suo dire sarebbe in grado di offrire grandi soddisfazioni sessuali a tutti, maschi e femmine, giovani e vecchi. Tutti ascoltano e molti ridono divertiti, però, mi sembra, nessuno compra.

Arrivati all'AMCES alle 16.30, in tutto nove ore e mezza, tre in meno che con l'autobus, però io sono ugualmente stanco.

Sistemate le mie cose nella stanza, breve incontro con Joseph e Baba per rivedere gli ultimi dettagli del corso e infine cena con Bienvenu e Olivier, nello stesso posto della volta scorsa. Questa sera cibo buono a prezzo africano (1,5 euro a testa).

Sono le 21, è prestissimo ma io ho sonno, e quindi saluto tutti e vado a dormire.

\section{Cotonou Venerdì 5 febbraio}

Primo giorno di questo $2^{\circ}$ corso: andato, e direi bene.

Tredici i partecipanti: quattro i medici, gli altri: tecnici di laboratorio e biologi. Anche due suore, una delle quali, suor Solange, durante una pausa mi si avvicina e dopo essersi presentata in francese incomincia a parlarmi in italiano. Vede il mio stupore e allora mi racconta che ha vissuto in Italia dieci anni per gli studi universitari, prima Biologia a Pisa e poi Medicina a Roma. Adesso lavora in un ospedale vicino a Lokossa, a due ore da qui, vicino al confine con il Togo. Mi dice che è contenta di essere tornata nel suo Paese ma che ha un po' di nostalgia dell'Italia.

Rispetto al corso di Tanguiéta, qui, meno domande e discussioni molto più brevi ed è per questo, credo, che abbiamo finito il programma con un'ora di anticipo. Joseph e Baba bene anche questa volta. Mi piace sentire come Joseph sa mettere l'accento sugli aspetti importanti e come Baba ci metta sempre qualche spunto originale, inaspettato e interessante.

Poco prima dell'inizio del corso, questa mattina, due sorprese:

- un WhatsApp da suor Florence di Materi, che mi manda i saluti suoi e delle consorelle. WhatsApp qui va alla grande, ce I'hanno in molti, anche suor Solange, e direi quasi il $100 \%$ dei partecipanti ai due corsi.

- Apparizione del tutto inaspettata, ma molto gradita, di Jacques, giovane nefrologo dell'ospedale universitario, che ha fatto una deviazione per venire a salutarmi e invitarmi a cena, questa sera a casa sua. Appuntamento alle 19 qui davanti, con Bruno, capo di Jacques, che dovrebbe passare a prendermi.
Ed è così che ho finito con l'aspettare, seduto di fianco al cancello dell'AMCES, che Bruno arrivasse, non alle 19 però, ma alle 21.30, con "sole" 2 ore e mezza di ritardo (ma siamo in Africa e ci sta).

E mentre aspettavo, è sceso un po' alla volta il buio, che qui è buio davvero, perché di luci pubbliche zero e private quasi. Le uniche luci quelle delle motorette, che a Cotonou sono migliaia e migliaia, e delle auto. Passano anche moltissime persone a piedi, le donne spesso con le loro mercanzie sulla testa. A un certo punto arriva lento lento un furgone da cui esce a tutto volume una bella musica, che prende, non solo me, ma anche il guardiano dell'AMCES che all'improvviso si alza dalla sedia e incomincia a ballare.

"Chi sono questi della musica?» gli chiedo «e cosa fanno?»

"Vendono i CD della cantante che stai sentendo.»

Nessuno compra, ma la musica continua e da una canzone si passa a un'altra, anche questa molto bella.

Ascolto, mi guardo attorno, osservo, e nel frattempo è arrivato anche un venticello fresco. Mi lascio trasportare dalle emozioni che questa via buia e trafficata, piena di gente e adesso anche di musica, suscita. E a certo punto mi ritrovo, un po' melanconico, a pensare: "Cosa possiamo capire noi di questo mondo cosi diverso e lontano dal nostro?".

Cena a casa di Jacques. Un po' alla periferia della città, sulla strada per Calavy. Una via non asfaltata, come quasi tutte qui, che sembra piuttosto sgarrupata. La casa di Jacques, però, è bella e di lusso, ben fatta. Jacques mi presenta sua moglie e i suoi due figli, un ragazzino di 13 anni e una bambina di 9. Ci sediamo e incominciamo a chiacchierare, Jacques ci offre un aperitivo. Dato che sono le 10, penso che tra un'ora al massimo ce ne andremo, un aperitivo, quattro chiacchiere e via. Naturalmente non va così, e alle 11 e mezza la moglie di Jacques ci invita a passare nella sala da pranzo perché "la cena è pronta".

Cena buonissima, compagnia anche, serata davvero piacevole, però all'una e quasi mezza eravamo ancora tutti lì, all'apparenza felici e contenti. lo, a questo punto, però, ho preso coraggio, mi sono alzato e chiedendo scusa ho detto che volevo andare a dormire perché all'indomani il corso sarebbe continuato. Non ha funzionato subitissimo, però alla fine ce l'ho fatta e alle 2 Bruno mi ha depositato davanti al cancello dell'AMCES.

Scherzi a parte, sono contento di essere andato a casa di Jacques, una persona che apprezzo molto, e di aver passato una bella serata, anche se temo che domani non sarò proprio sveglissimo.

Ma a parte questo, sulla via del ritorno sono stato molto colpito nel vedere che a quell'ora, nel buio più completo, c'erano ancora in giro donne - e con loro anche una bambina che avrà avuto sì e no 10 anni - che camminavano lungo la strada, tutte con i loro carichi sulla testa. Stupito, ho chiesto a Bruno chi potessero essere.

"Venditrici, che tornano a casa dopo la loro giornata di lavoro.» 
«Scusa Bruno, ma qui la giornata di lavoro finisce alle 2 del mattino?!»

«Per queste donne sì!»

Alle 2 del mattino, a piedi verso casa cariche come somari, e con loro una bambina ...

E così mentre gli occhi fanno sempre più fatica a stare aperti e mi sto per addormentare, il cuore non può fare a meno di tornare indietro, lungo la strada, a camminare anche lui con quelle donne e quella bambina.

\section{Cotonou Sabato 6 febbraio}

Mattina. Dormito dalle 2 alle 6.30: troppo poco. Jacques, io ti ringrazio molto per la bella serata, ma cosa ti è venuto in mente di cominciare la cena quasi a mezzanotte e di continuare a tirar fuori bottiglie di liquori e champagne, una dopo l'altra? Non ti sei accorto che io bevevo solo acqua e che avevo una faccia da sonno già quando sono arrivato? Ok, alla fine eravamo tutti contenti, però ti rendi conto che se io non mi fossi alzato dicendo che dovevo tornare a casa per il corso saremmo ancora tutti lì, a casa tua? E dimmi, Jacques, saresti stato contento di averci ancora lì alle 6.30 della mattina?

Sera. Ok, anche questa seconda e ultima giornata del corso è andata.

Come già a Tanguiéta, il livello di conoscenza è basso, l'esperienza poca, ma la voglia di migliorare apparentemente tanta. Domande deboli e discussioni brevi, ma esercizi di refertazione super e un sacco di empatia. Insomma, so bene da dove si parte e lo sanno anche loro. L'importante è far arrivare il messaggio che si può fare molto di più anche con $\mathrm{i}$ pochi mezzi a disposizione e a costo zero. Ciò che fa la differenza è avere voglia di migliorare e di applicarsi, con metodo e costanza, e niente di più.

Dopo il corso e fino all'ora di cena sono con Joseph e Baba sul terrazzo a stendere il rapporto dei due corsi da inviare all'AMCES e a mettere giù il programma preliminare per il corso teorico-pratico di una settimana che vorremmo fare a novembre a Tanguiéta per i Paesi dell'Africa Francofona. Ragazzi, ormai ci sentiamo rodati e alziamo l'asticella.

Finito di lavorare, l'idea è di andare a cena insieme, così Joseph e io ci troviamo davanti al cancello dell'AMCES ad aspettare Baba, che nel frattempo è andato a farsi risuolare $\mathrm{i}$ sandali da un ciabattino qui vicino. Quando ricompare, però, Baba ci dice che lui la cena l'ha già fatta, per conto suo.

Lo guardo un po' stupito, anche se dentro di me immagino cosa possa essere successo: ha trovato per strada un posto che costa meno rispetto a dove volevamo andare tutti insieme e si è fermato lì.

«Ok, Baba, ci sta, però venirlo a dire anche a noi, no? Potevamo spendere meno anche Joseph e io e intanto potevamo stare insieme, non ti pare?»

Baba mi ascolta, fa cenno di sì con la testa e mi dà ragione, ma mi guarda come per dire "ormai è fatta". Guardo di nuovo

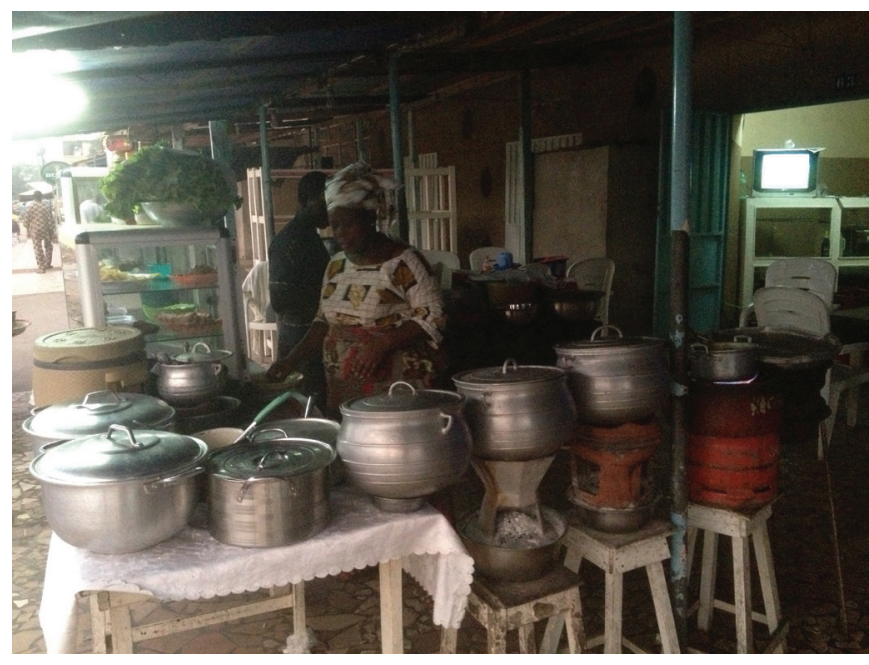

Fig. 8 - II nostro ristorante africano vicino all'AMCES.

Baba negli occhi, e poi mi giro verso Joseph: io guardo lui e lui guarda me e poi sottovoce mi sussurra: «Te l'avevo detto o no che Baba è un po' arcaico?».

Ok Joseph, posso essere d'accordo con te, ma del resto cosa c'è di più facile che essere "un po' arcaico" in Africa?

Comunque, dopo qualche minuto finiamo nel solito ristorante (Fig. 8), dove in due ceniamo e in tre chiacchieriamo.

\section{Cotonou Domenica 7 febbraio}

Mi sono alzato presto per salutare Joseph e Baba che sono partiti alla volta di Tanguiéta, di nuovo con la vettura dell'ospedale guidata da Roger.

Joseph e Baba: due persone con cui mi trovo bene e che quando partono mi mancano. Per me l'Africa è tante cose, non ultima le amicizie.

Poi, giornata tranquilla tutta all'ombra dell'AMCES e dintorni (sta a vedere che mi affeziono anche a questa via).

Nel pomeriggio appuntamento con Jacqueline, la vedova di Guy, e suo figlio Jauriac, che ancora non conoscevo. Abbiamo chiacchierato piacevolmente e amichevolmente per quasi due ore, e ci siamo salutati con la promessa di sentirci ogni tanto via WhatsApp.

E sempre a proposito di WhatsApp, alle 17.30 messaggio da Joseph: "arrivati sani e salvi a Tanguiéta".

\section{Cotonou Lunedi 8 febbraio}

Ultimo giorno. Da segnare a colori e in grassetto per l'acquisto dello scooter per Bienvenu, una "Haojué 110" di produzione indiana, nuova di pacca e rosso fiammante al prezzo "non negoziabile" - ci tiene a dirmi fin dall'inizio il venditore di 470.000 franchi CFA (= $717 €)$, ma con casco integrale e impermeabile - che qui nella stagione delle piogge serve davvero - inclusi. 
Bienvenu è molto contento: desiderava una moto da tempo per potersi spostare avanti e indietro da casa all'università, al centro di dialisi, e ai vari posti in cui trova di tanto in tanto qualche lavoro da fare. Per il nostro gruppo "Noi di Tanguiéta" non è stata una scelta facile acconsentire a questa richiesta, perché non capivamo quanto fosse solo un capriccio e quanto invece fosse davvero una necessità. Dopo uno scambio di opinioni con Alberto e Ondina, Margherita, Nini, Simona e altri alla fine è prevalsa l'idea di comprarla, questa benedetta moto, e di dare un po' di fiducia oltre che di felicità a questo nostro Bienvenu, malato e lontano dalla sua famiglia.

E così, dopo un giro in banca per cambiare gli euro in franchi CFA e una mezz'oretta di attesa per la messa a punto della moto siamo finalmente partiti, Bienvenu alla guida e io dietro - con un po' di strizza - alla volta dell'ospedale universitario. Dove siamo arrivati in pochi minuti e da dove Bienvenu, il mio nuovo autista personale, è subito ripartito alla volta di casa a far vedere la moto a Olivier, mentre io mi infilavo in ospedale. Prima nel laboratorio di Augustin per cercare di capire se ci arriverà mai l'esame del sedimento urinario (nonostante i due mesi passati da Augustin nel nostro laboratorio al Croff e il nuovo microscopio che gli è appena arrivato), e poi nell'ufficio di Jacques per un aggiornamento sulla Nefrologia in Bénin.

Alla fine ho telefonato a Bienvenu, che è venuto a prendermi, con la moto ovviamente, per portarmi all'AMCES, dove siamo stati raggiunti da Olivier e Martin, per il pranzo di saluto, al solito posto, dove si mangia bene e si spende poco (particolare non del tutto secondario perché lì chi paga è sempre il "bianco", cioè io ...).

E infine, con l'auto di Henry all'aeroporto, con Bienvenu e Olivier in moto dietro di noi (Fig. 9).

\section{Milano Martedi 9 febbraio}

Mattina. Sono arrivato a casa, dove abita il cuore. Ma nel cuore ho anche l'Africa e le persone che ho incontrato, quelle che conosco e quelle che non conosco, il cui sguardo ho incrociato anche per un solo secondo e solo per caso.

Sono un po' rintronato, ma per forza, in aereo io non riesco proprio a dormire: abbasso lo schienale del sedile, allungo le gambe, le accavallo, le piego, le allungo di nuovo, mi giro e mi rigiro senza un attimo di tregua. Niente da fare, non ci riesco, nemmeno con il Tavor, mentre tutti gli altri attorno a me dormono alla grande e qualcuno, beato, perfino russa ...

\section{Epilogo}

Un WhatsApp inviatomi da Pauline a fine febbraio mi informa che pochi giorni dopo la mia partenza Daniel è stato

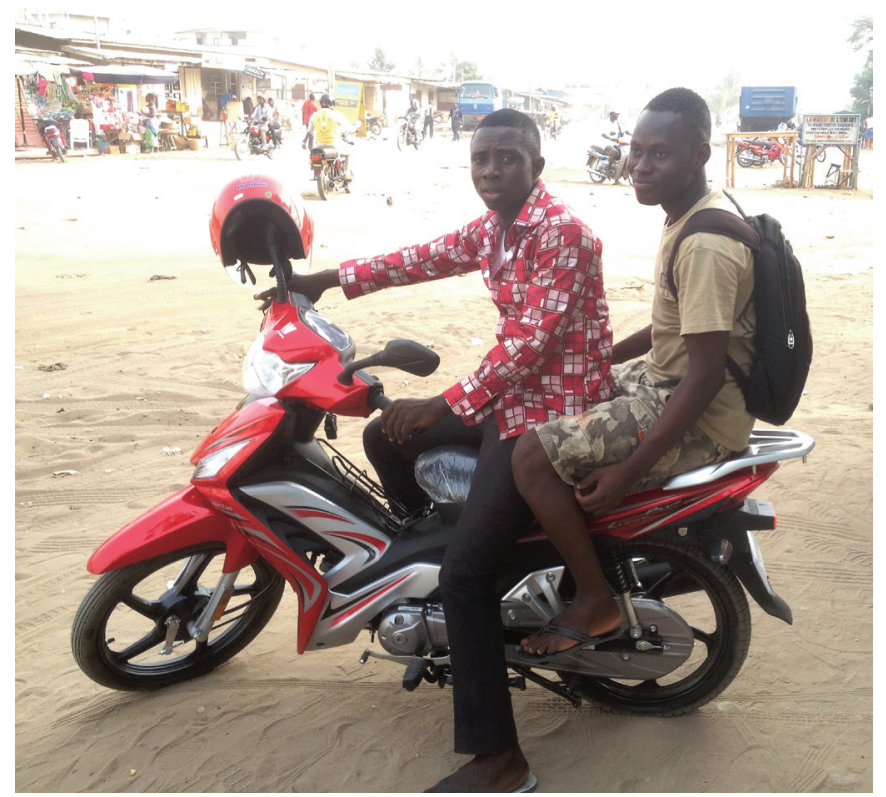

Fig. 9 - Bienvenu e Olivier sul nuovo scooter.

dimesso dall'ospedale senza alcuna speranza di guarigione e che non si è presentato al controllo ambulatoriale che gli era stato fissato.

Una mail inviatami da suor Martine ai primi di luglio mi informa che Penagui è morta in ospedale il 21 giugno e che subito dopo sua mamma è ritornata, con gli altri due bambini, nella sua casa nel villaggio vicino a Materi.

Queste due notizie, anche se prevedibili, mi addolorano moltissimo. Non posso non pensare che se Daniel e Penagui fossero nati non in Africa, ma in Italia, a quest'ora sarebbero ancora vivi e curati in modo adeguato e gratuitamente e con la prospettiva di essere sottoposti a un trapianto di rene che li riporterebbe a una vita pressoché normale. Posso quindi non pensare a quanta ingiustizia esiste ancora nel mondo e a quanto noi qui, nonostante le nostre continue lamentele, siamo fortunati?

\section{Bibliografia}

1. Fogazzi T. Dove 2+2 non fa 4. Lecce: Manni Editori 2014. Questo libro raccoglie i diari delle missioni nefrologiche dell'autore dal 2002 al 2012.

2. Mastracola P. L'esercito delle cose inutili. Torino: Einaudi 2015.

3. Schweitzer A. À l'orée de la forêt vièrge. Lausanne: Edition La Concorde 1923. 